\title{
TNF-a blockade is ineffective in animal models of established polycystic kidney disease
}

\author{
Jeffrey Roix and Saurabh Saha*
}

\begin{abstract}
Background: Given the large medical burden of polycystic kidney disease (PKD) and recent clinical trial failures, there is a need for novel, safe and effective treatments for the disorder.

Methods: In PCK rat and PKD2 ${ }^{(\text {ws } 25 / \text { w183) }}$ mouse models, entanercept was administered once every three days at 5 or $10 \mathrm{mg} / \mathrm{kg}$, once daily. Mozavaptan was administered as a pilot control, provided continuously via milled chow at $0.1 \%$. Animals were assessed for measures of pharmacodynamic response, and improvements in measures of polycystic kidney disease.

Results: Entanercept treatment modulated inflammatory markers, but provided limited therapeutic benefit in multiple animal models of established polycystic kidney disease. Kidney weight, cyst burden and renal function markers remained unchanged following administration of etanercept at various dose levels and multiple treatment durations.

Conclusions: While it remains possible that TNF-a inhibition may be effective in truly preventative settings, our observations suggest this pathway is less likely to exhibit therapeutic or disease-modifying efficacy following the standard clinical diagnosis of disease.
\end{abstract}

Keywords: Polycystic kidney disease, Etanercept, TNF-a, PKHD1, PKD2

\section{Background}

As many as 1 in 20 patients on dialysis suffer from polycystic kidney disease: this chronic, progressive disorder emerges over years in patients who carry a spectrum of well-characterized genetic alterations [1]. Autosomal dominant and recessive mutations in the polycystin gene family (PKHD1, PKD2) are thought to cause an array of defects in basic renal epithelial cell function, which in turn culminate in the hallmark cysts and kidney dysfunctions characteristic of the disease $[2,3]$.

Despite an understanding of both pathophysiologic and correlative molecular genetic processes, effective and safe treatments for PKD are not currently available, and recent interventional trials for the disease have been disappointing. Preclinical studies highlighted the possible value of targeting the vasopressin and mTOR/AKT pathways [4-6]. Several PKD clinical trials assessing drugs that target these

\footnotetext{
* Correspondence: ssaha@biomed-valley.com

Biomed Valley Discoveries Inc, 4520 Main Street, Suite 1650, MO 64111, Kansas City, USA
}

\section{Biomed Central}

(c) 2013 Roix and Saha; licensee BioMed Central Ltd. This is an open access article distributed under the terms of the Creative Commons Attribution License (http://creativecommons.org/licenses/by/2.0), which permits unrestricted use, distribution, and reproduction in any medium, provided the original work is properly cited. in the face of appreciable side effects [7-9].

In this setting, it is promising that studies of basic biological processes associated with PKD pathophysiology have yielded new therapeutic targets. TNF- $\alpha$ is a canonical immune cytokine that activates inflammatory signaling in disorders ranging from arthritis to bowel disease $[10,11]$. Recently, this pathway was implicated in fundamental cellular processes of PKD: active TNF- $\alpha$ interferes with processing and membrane presentation of PKD2, effectively phenocopying genetic ablation of the disease locus. TNF- $\alpha$ was shown to be a both necessary and sufficient modifier of polycystic kidney disease. Intriguingly, TNF- $\alpha$ inhibition, using the approved biologic drug etanercept, could prevent kidney cyst formation in animals heterozygous for PKD2 deficiency that are normally predisposed to the disease [12].

While the genetic basis of PKD is understood, definitive clinical diagnosis occurs only after the evolution of established disease, when ultrasound is used to confirm the presence of renal cysts [13]. Therefore, we sought to 
translate the promising activity of etanercept in additional rodent genetic models where cysts are present at the onset of intervention.

\section{Methods \\ Animals}

A rat colony homozygous for mutation at the Pkhd1 locus (allele: PcK/Crljcrl-Pkhd1 ${ }^{\text {pck }}$ ) was obtained from Charles River (Wilmington, MA). Male rats were employed in drug studies. Identification and characterization of this spontaneous mutant colony has been described [14]. A colony of PKD2 ws25/w183 compound heterozygous mice (referred to herein as ws25/-) kindly provided by Albert Einstein College of Medicine, and re-derived and maintained. Mice were genotyped by southern blots, and generally an equal number of male and female animals were apportioned to each study group. The Taconic Animal Care and Use Committee approved all study protocols. Animals had free access to water and standard diets.

\section{Experimental protocols}

A summary of treatment and study design is included in Table 1. Rats were obtained at three weeks of age, and acclimated for one week preceding treatment. Etanercept (Enbrel, Amgen) was reconstituted regularly (vehicle 0.9\% benzyl alcohol in sterile water) and single aliquots were used for a maximum of 4 days; dosing route was intraperitoneal (i.p.). Vehicle treatments were designed to match the excipient composition: $200 \mathrm{mM}$ mannitol, $30 \mathrm{mM}$ sucrose, $1 \mathrm{mM}$ tromethamine in sterile water, pH7.4. Mozavaptan was synthesized (Focus Synthesis, San Diego, CA) and administered on a continuous basis via standard diet milled at the indicated concentrations on a perweight basis. At the end of treatment, animals were anesthetized by carbon dioxide inhalation. Kidneys were removed and weighed; blood and serum was obtained by cardiac puncture method. The left kidneys were weighed and then fixed in 10\% neutral-buffered formalin for 48Hrs, processing for histologic examinations; right kidneys were weighed and snap-frozen in dry ice for biomarker protein assay work.

Kidneys were embedded, sectioned and stained with hematoxylin and eosin (H\&E) for histopathological evaluation of the degrees and severity of cyst formation. Tissues were sectioned longitudinally through the center keep the location of assessments constant. Cyst number was assessed by gross inspection and count from 3 random fields $(\times 40$ magnification) per animal, and averaged across cohorts.

Mice were weaned and allowed to acclimate to either 4 or 13 weeks of age before treatment began. Drug treatments and subsequent tissue, blood and any biomarker analyses were performed as described above.

In rats, water intake was assessed by periodically weighing cage bottles, and urine output was measured following forced manual voiding. In mice, urine production was assessed using metabolic cage monitoring; recording sessions were acquired in two separate morning and evening sessions, for two hours each. Mouse water consumption was monitored through periodic cage bottle weighing.

\section{Kinase signaling analysis}

Kinase signaling was assessed on homogenized kidney lysates using a multiplex, bead-based suspension protein array service (AssayGate Inc, Ijamsville, MD).

\section{Serum chemistry}

Serum urea nitrogen levels and creatinine were measured using a Beckman autoanalyzer and standard colorimetric assessments (Beckman Instruments, Fullerton, CA).

\section{Statistical analyses}

Multiple group comparisons were performed using a one-way ANOVA with post-test according to Dunnet. $\mathrm{P}<0.05$ was considered statistically significant. Values are expressed as means \pm SEM.

\section{Results and discussion}

Initially, we studied the pck rat disease model, which harbors homozygous null mutations in the orthologous PKHD1 susceptibility locus: this model of autosomal recessive PKD (ARPKD) was previously used to study the potential therapeutic effects of vasopressin and mTOR/ AKT pathway inhibitors $[4,15]$. We first established an optimal dosing protocol for etanercept, as the drug could display unexpected pharmacokinetic and pharmacodynamic effects in certain rodent models. These pilot studies showed that, in kidneys analyzed following 4 weeks of treatment, as little as $0.5 \mathrm{mg} / \mathrm{kg}$ etanercept dosed every three days was sufficient to markedly reduce both NF-kB (p65-S536) and p38 stress kinase pathway signaling, which are established mechanistic biomarkers of TNF-a signaling [16] (Figure 1A).

Having proven that etanercept could reduce relevant inflammatory signaling in the kidneys of pck rats, we next studied whether the drug could improve established markers of renal function and PKD pathology. In 4-week old animals treated for one month, gross kidney weights, as well as average cyst number quantified after histological examination, were unchanged by etanercept doses as high as $10 \mathrm{mg} / \mathrm{kg}$ (Figure 1B). Similarly, the renal function markers blood urea nitrogen (BUN) and creatinine remained unchanged following treatment (Figure 1B). Other investigators have noted that rodent models of PKD are sensitive to the timing and duration of treatment [6]. Therefore, we tested prolonged etanercept dosing at $5 \mathrm{mg} / \mathrm{kg}$ every 3 days for 8 weeks; in this background, we additionally tested continuous administration of the vasopressin antagonist mozavaptan at 
Table 1 Treatment and methods summary for animal studies

\begin{tabular}{|c|c|c|c|c|c|c|c|c|}
\hline Study & Genotype & Age at treatment & Animals per arm & Treatments & Administration route & Dose & Schedule & Treatment duration \\
\hline \multirow{2}{*}{$\begin{array}{l}\text { ARPKD rat, short-term } \\
\text { therapeutic intervention }\end{array}$} & PcK/Crljcrl-Pkhd1pck & 4 weeks & 10 & Vehicle & NA & NA & NA & 4 weeks \\
\hline & PcK/Crljcrl-Pkhd1pck & 4 weeks & 10 & Mozavaptan & i.p. & $0.5,5,10 \mathrm{mg} / \mathrm{kg}$ & Once, every third day & 4 weeks \\
\hline \multirow{3}{*}{$\begin{array}{l}\text { ARPKD rat, Long-term } \\
\text { therapeutic intervention }\end{array}$} & PcK/Crljcrl-Pkhd1pck & 4 weeks & 10 & Vehicle & NA & NA & NA & 8 weeks \\
\hline & PcK/Crljcrl-Pkhd1pck & 4 weeks & 10 & Mozavaptan & Milled chow & $0.10 \%$ & ad libitum & 8 weeks \\
\hline & PcK/Crljcrl-Pkhd1 pck & 4 weeks & 10 & Etanercept & i.p. & $5 \mathrm{mg} / \mathrm{kg}$ & Once, every third day & 8 weeks \\
\hline \multirow{3}{*}{$\begin{array}{l}\text { ADPKD (PDK2 ws25/-) mouse, } \\
\text { therapeutic intervention }\end{array}$} & PKD2 ws25/- & 12 weeks & 14 & Vehicle & NA & NA & NA & 8 weeks \\
\hline & PKD2 ws25/- & 12 weeks & 11 & Mozavaptan & Milled chow & $0.10 \%$ & ad libitum & 8 weeks \\
\hline & PKD2 ws25/- & 12 weeks & 15 & Etanercept & i.p. & $5 \mathrm{mg} / \mathrm{kg}$ & Once, every third day & 8 weeks \\
\hline \multirow{3}{*}{$\begin{array}{l}\text { ADPKD (PDK2 ws25/-) mouse, } \\
\text { prophylactic intervention }\end{array}$} & PKD2 ws25/- & 4 weeks & 15 & Vehicle & NA & NA & NA & 8 weeks \\
\hline & PKD2 ws25/- & 4 weeks & 15 & Mozavaptan & Milled chow & $0.05 \%$ & ad libitum & 8 weeks \\
\hline & PKD2 ws25/- & 4 weeks & 15 & Etanercept & i.p. & $5,10 \mathrm{mg} / \mathrm{kg}$ & Once, every third day & 8 weeks \\
\hline
\end{tabular}



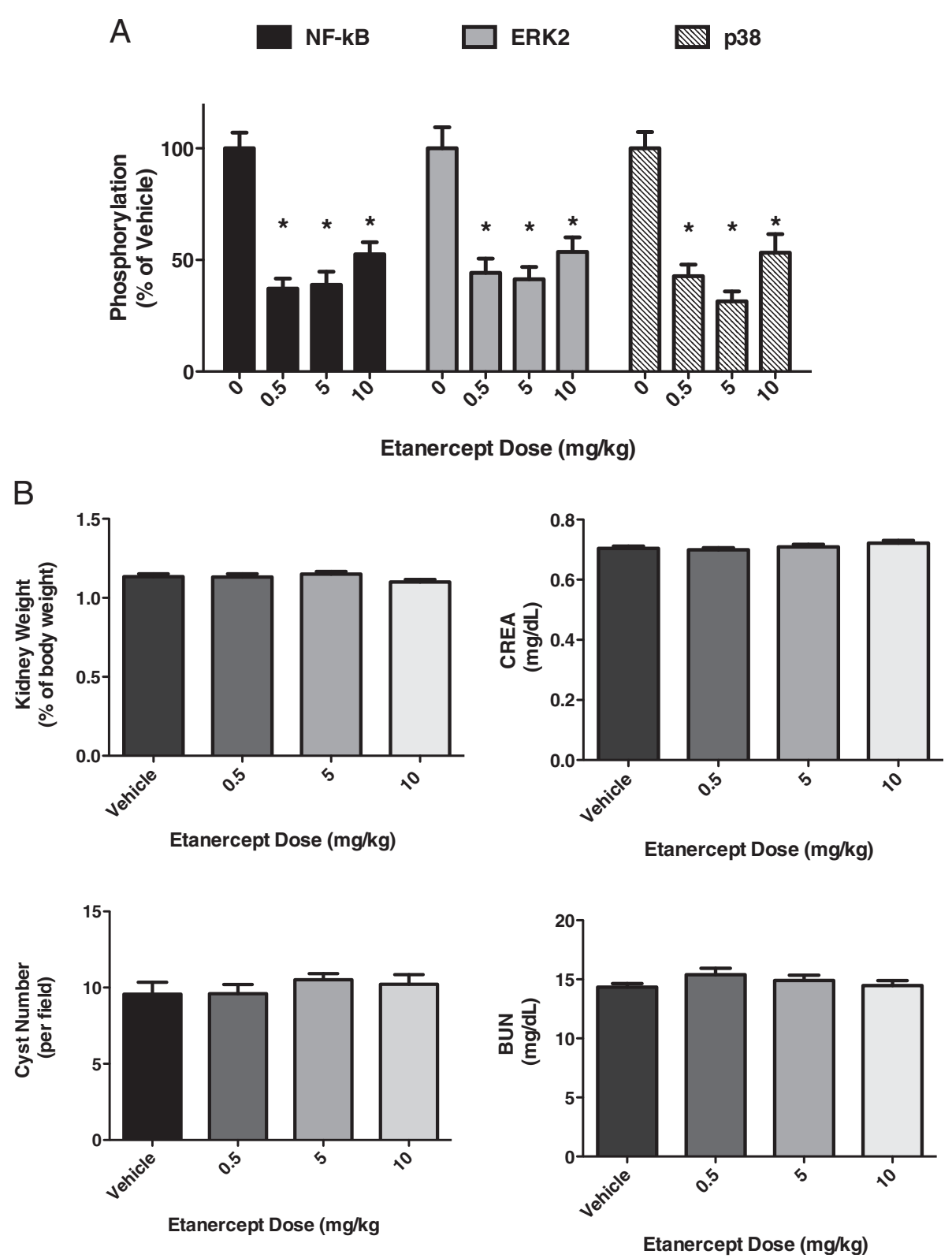

Figure 1 Short-term (4 weeks) entanercept pharmacodynamics and efficacy in pck rats. (A) Stress kinase phosphorylation from kidney tissue lysates $(\mathrm{N}=4)$ is significantly reduced following 4 weeks dosing with etanercept at various levels. (B) Kidney weight, cyst number, serum urea nitrogen and creatinine are unchanged following etanercept treatment for 4 weeks $(N=10)$.

pharmacologically relevant doses. We noted that mozavaptan induced aquaretic effects, which confirmed that our studies could properly recapitulate known pharmacologic effects of this drug class. Still, in the more chronic treatment setting, neither etanercept nor mozavaptan proved efficacious against multiple therapeutic markers of PKD. Surprisingly, both gross kidney weight and cyst number were moderately increased in both drug treatment arms (Table 2). While BUN was largely unchanged following treatment, animals treated with either etanercept or mozavaptan had blood creatinine levels greater than untreated animals (Table 2). Given the previously observed effects of vasopressin antagonists in preserving kidney volume and reducing cysts $[4,6]$, though, we conclude etanercept is largely ineffective in this model of ARPKD.

Still, it remained possible etanercept could be effective in models of autosomal dominant PKD (ADPKD). Here, we studied mice hemizygous for the PKD2 ws25 allele; this latent disease model rapidly develops cysts in a stochastic fashion, following somatic recombination and loss-of-heterozygosity [17]. We first tested etanercept in 13 week-old PKD2 ws25/- animals, which have previously 
Table 2 Efficacy of long-term (8 week) treatment with mozavaptan or etanercept in pck rats ${ }^{\mathrm{a}}$

\begin{tabular}{cccccc}
\hline Treatment & $\begin{array}{c}\text { Kidney weight } \\
\text { (\% of bw) }\end{array}$ & $\begin{array}{c}\text { Cyst number } \\
\text { (per field) }\end{array}$ & BUN (mg/dL) & CREA (mg/dL) & $\begin{array}{c}\text { Water consumption }{ }^{\mathbf{d}} \\
\text { (mL/rat/day0029) }\end{array}$ \\
\hline Vehicle & $1.05 \pm 0.023$ & $9.2 \pm 0.71$ & $14.9 \pm 0.51$ & $0.63 \pm 0.05$ & $42.96 \pm 1.96$ \\
Mozavaptan $(0.1 \%)$ & $1.12 \pm 0.04$ & $12.1 \pm 1.4$ & $16.2 \pm 0.39$ & $0.71 \pm 0.03^{\mathrm{b}}$ & $63.88 \pm 0.56^{\mathrm{c}}$ \\
Etanercept $(5 \mathrm{mg} / \mathrm{kg})$ & $1.16 \pm 0.036$ & $12 \pm 1.3$ & $16.4 \pm 0.4$ & $0.71 \pm 0.06^{\mathrm{b}}$ & $38.95 \pm 1.40$ \\
\hline
\end{tabular}

${ }^{\mathrm{a}}$ Data shown are group average $\pm \mathrm{SEM}, \mathrm{N}=10$.

${ }^{\mathrm{b}}$ Comparison between vehicle and treatment group(s), $\mathrm{p}<0.05$.

${ }^{c}$ Comparison between vehicle and treatment group $(\mathrm{s}), \mathrm{p}<0.001$.

${ }^{\mathrm{d}}$ Water consumption measured over final week of treatment.

been shown to harbor overt signs consistent with polycystic kidney disease. Following dosing for 8 weeks, $5 \mathrm{mg} / \mathrm{kg}$ etanercept did not improve kidney weight, cyst number, or BUN. As in pck rats, we also administered $0.1 \%$ mozavaptan continuously via diet; perhaps surprisingly, this treatment also proved ineffective across the multiple parameters investigated (Table 3 ).

Finally, we studied etanercept initiated at the earliest time point achievable in in-vivo settings. Starting at 4 weeks of age, we administered either 5 or $10 \mathrm{mg} / \mathrm{kg}$ etanercept to PDK2 ws25/- mice once every three days for two months. As in pck rats, etanercept proved ineffective in treating various aspects of PKD: kidney weight, and cyst number were not reduced following treatment, and BUN also remained unchanged (Table 3). In total, we conclude that, in settings that would reveal either therapeutic or disease-modifying activities, etanercept is largely ineffective at treating the key pathologic and physiologic dysfunctions of PKD.

\section{Conclusions}

Considering our data in the context of prior mechanistic and clinical studies, we suggest several key conclusions. First, and most importantly, etanercept, and likely TNF- $\alpha$ blockade in general, appears to be a relatively poor candidate therapeutic approach for study in future clinical trials. Clinical trials of vasopressin and mTOR antagonists have shown marginal efficacy and important side effects, despite showing promising preclinical efficacy for several of our investigated parameters, in rodent models closely related those employed here. Our studies were powered to discern therapeutic effects similar in magnitude as those drugs currently in clinical testing. Therefore, TNF- $\alpha$ blockade seems less attractive compared to other candidate therapeutic mechanisms both currently and prospectively being assessed for clinical translation.

Second, our study underscores how fundamental molecular and cellular events observed in preclinical research can be difficult to translate into treatments for disease biology as it presents during common clinical practice. Current clinical criteria dictate that, even in the presence of strongly predictive genetic risk, formal diagnosis of ADPKD requires the observation of cysts via ultrasound exam [13]. By contrast, $\mathrm{Li}$ et al. showed that, in young mice heterozygous for PKD2 loss, cyst formation could be prevented by prophylactic etanercept [12]. While promising, the analogous application of this observation into clinical practice would imply life-long dosing and consequent immunosuppression, initiated in very young patients with a confirmed genetic background of PKD. Preventative therapy along these lines would likely be difficult to test in clinical trials, and could also prove challenging in everyday clinical practice.

Lastly, our data suggest additional, relevant hypotheses concerning disease pathology and treatment in PKD. Patients presenting with PKD are likely phenotypically null for essential polycystin gene function(s)-single PKHD1 or "two-hit" PKD2 mutations both damage renal cell function

Table 3 Prophylactic and therapeutic administration of mozavaptan or etanercept in PKD2( ${ }^{\text {ws25/-) }}$ mice $^{\text {a }}$

\begin{tabular}{|c|c|c|c|c|c|c|c|}
\hline Treatment & $\begin{array}{c}\text { Age at } \\
\text { treatment }\end{array}$ & $\mathbf{N}$ & $\begin{array}{l}\text { Kidney weight } \\
\text { (\% of bw) }\end{array}$ & $\begin{array}{l}\text { Cyst number } \\
\text { (per field) }\end{array}$ & $\begin{array}{c}\text { BUN } \\
(\mathrm{mg} / \mathrm{dL})\end{array}$ & $\begin{array}{l}\text { Urine production } \\
\text { (mL/mouse) }\end{array}$ & $\begin{array}{l}\text { Water consumption } \\
\text { (mL/mouse/day) }\end{array}$ \\
\hline Vehicle & 13 weeks & 14 & $1.85 \pm 0.17$ & $6.7 \pm 0.7$ & $20.6 \pm 1.2$ & $0.99 \pm 0.20$ & $4.0 \pm 0.04$ \\
\hline Mozavaptan (0.1\%) & 13 weeks & 11 & $1.74 \pm 0.11$ & $6.9 \pm 1.1$ & $23.6 \pm 2.0$ & $4.93 \pm 1.64^{c}$ & $11.1 \pm 0.35^{c}$ \\
\hline Etanercept (5 mg/kg) & 13 weeks & 15 & $1.75 \pm 0.09$ & $6.5 \pm 0.8$ & $20.1 \pm 1.5$ & NA & $4.0 \pm 0.06$ \\
\hline Vehicle & 4 weeks & 15 & $1.96 \pm 0.15$ & $7.0 \pm 0.7$ & $24.8 \pm 2.7$ & NA & $6.1 \pm 0.18$ \\
\hline Mozavaptan (0.05\%) & 4 weeks & 15 & $1.78 \pm 0.07$ & $7.7 \pm 0.8$ & $26.1 \pm 2.1$ & NA & $9.6 \pm 0.35^{c}$ \\
\hline Etanercept (10 mg/kg) & 4 weeks & 15 & $1.84 \pm 0.2$ & $5.5 \pm 0.6$ & $21.9 \pm 1.6$ & NA & $5.9 \pm 0.16$ \\
\hline Etanercept (5 mg/kg) & 4 weeks & 15 & $1.77 \pm 0.17$ & $6.2 \pm 0.7$ & $23.2 \pm 2.0$ & NA & $5.8 \pm 0.18$ \\
\hline
\end{tabular}

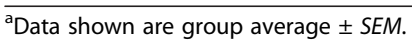

bUrine production over 4 hours monitoring in metabolic cage; separate measure of daily water consumption, by bottle weighing

${ }^{\mathrm{c} C}$ Comparison between vehicle and treatment group, $\mathrm{p}<0.05$. 
sufficiently to cause disease. While Li et al. suggested that TNF- $\alpha$ blockade could rescue residual PKD2 activity, our work suggests that later pathophysiological events in PKD are largely TNF- $\alpha$ independent. Also, as TNF- $\alpha$ has been long appreciated as a key, nodal point of inflammatory signaling, it seems possible that other anti-inflammatory approaches may also prove ineffective in PKD-specifically, approaches that simply decrease NF-kB or p38 stress kinase signaling (as in our studies) may not sufficiently modify the underlying pathophysiology of PKD.

In summary, our studies have explored the possible therapeutic benefit of TNF- $\alpha$ blockade in rodent models of PKD previously used to nominate agents for interventional clinical trials. We suggest our data de-prioritize this putative disease mechanism for future clinical testing in settings of established disease. It remains possible, however, that alternate anti-inflammatory approaches could be safe and effective treatments for PKD, and that chronic TNF- $\alpha$ inhibition could prevent the emergence of PKD in patients who are genetically "at-risk", but harbor occult disease.

\section{Competing interest}

The authors declare that they have no competing interest.

\section{Authors' contributions}

SS designed the experimental approach and studies, and coordinated experimental execution. JR analyzed data. JR and SS wrote the manuscript. Both authors read and approved the final manuscript.

\section{Acknowledgements}

The authors thank Dr. Reza Halse and Dr. John Couse for assistance in experimental design and execution. We thank Stefan Somlo M.D. for advice and providing mouse models. We also thank Rong Li PhD, Vicente Torres M. D., Dr Mason Freeman M.D. and for advice and consultation.

Received: 28 June 2013 Accepted: 14 October 2013

Published: 25 October 2013

\section{References}

1. Grantham JJ: Clinical practice Autosomal dominant polycystic kidney disease. N Engl J Med 2008, 359(14):1477-1485.

2. Delmas P: Polycystins: from mechanosensation to gene regulation. Cell 2004, 118(2):145-148.

3. Weimbs T: Polycystic kidney disease and renal injury repair: common pathways, fluid flow, and the function of polycystin-1. Am J Physiol Renal Physiol 2007, 293(5):F1423-F1432.

4. Wang X, Gattone V, Harris PC, Torres VE: Effectiveness of vasopressin V2 receptor antagonists OPC-31260 and OPC-41061 on polycystic kidney disease development in the PCK rat. J Am Soc Nephrol 2005, 16(4):846-851.

5. Wahl PR, Serra AL, Le Hir M, Molle KD, Hall MN, Wüthrich RP: Inhibition of mTOR with sirolimus slows disease progression in Han:SPRD rats with autosomal dominant polycystic kidney disease (ADPKD). Nephrol Dial Transplant 2006, 21(3):598-604.

6. Meijer E, Gansevoort RT, de Jong PE, et al: Therapeutic potential of vasopressin $\mathrm{V} 2$ receptor antagonist in a mouse model for autosomal dominant polycystic kidney disease: optimal timing and dosing of the drug. Nephrol Dial Transplant 2011, 26(8):2445-2453.

7. Watnick T, Germino GG: mTOR inhibitors in polycystic kidney disease. N Engl J Med 2010, 363(9):879-881.

8. Schrier RW: Randomized intervention studies in human polycystic kidney and liver disease. J Am Soc Nephrol 2010, 21(6):891-893.

9. Serra AL, Poster D, Kistler AD, et al: Sirolimus and kidney growth in autosomal dominant polycystic kidney disease. N Engl J Med 2010 363(9):820-829.
10. Scott DL, Kingsley GH: Tumor necrosis factor inhibitors for rheumatoid arthritis. N Engl J Med 2006, 355(7):704-712.

11. Danese S, Fiocchi C: Ulcerative colitis. N Engl J Med 2011, 365(18):1713-1725.

12. Li X, Magenheimer BS, Xia S, et al: A tumor necrosis factor-alpha-mediated pathway promoting autosomal dominant polycystic kidney disease. Nat Med 2008, 14(8):863-868.

13. Pei Y, Obaji J, Dupuis A, et al: Unified criteria for ultrasonographic diagnosis of ADPKD. J Am Soc Nephrol 2009, 20(1):205-212.

14. Katsuyama M, Masuyama T, Komura I, Hibino T, Takahashi H: Characterization of a novel polycystic kidney rat model with accompanying polycystic liver. Exp Anim 2000, 49(1):51-55.

15. Renken C, Fischer DC, Kundt G, Gretz N, Haffner D: Inhibition of mTOR with sirolimus does not attenuate progression of liver and kidney disease in PCK rats. Nephrol Dial Transplant 2011, 26(1):92-100.

16. Chen G, Goeddel DV: TNF-R1 signaling: a beautiful pathway. Science 2002, 296(5573):1634-1635.

17. Wu G, D'Agati V, Cai Y, et al: Somatic inactivation of Pkd2 results in polycystic kidney disease. Cell 1998, 93(2):177-188.

doi:10.1186/1471-2369-14-233

Cite this article as: Roix and Saha: TNF-a blockade is ineffective in animal models of established polycystic kidney disease. BMC Nephrology 2013 14:233.

\section{Submit your next manuscript to BioMed Central and take full advantage of:}

- Convenient online submission

- Thorough peer review

- No space constraints or color figure charges

- Immediate publication on acceptance

- Inclusion in PubMed, CAS, Scopus and Google Scholar

- Research which is freely available for redistribution 